\title{
Diffusive Representation and Sliding Mode Control of Charge Trapping in $\mathrm{Al}_{2} \mathrm{O}_{3}$ MOS Capacitors
}

\begin{abstract}
The objective of this paper is to introduce a modeling strategy to characterize the dynamics of the charge trapped in the dielectric of MOS capacitors, using Diffusive Representation. Experimental corroboration is presented with MOS capacitors made of Alumina in three different scenarios. First, the model predictions are compared with the trapped charge evolution due to arbitrary voltage excitations. Second, the predictions are compared with the measurements of a device in which a sigmadelta control of trapped charge is implemented. Finally, the time evolution when the device is simultaneously controlled and irradiated with $\mathrm{X}$-rays is compared with the predictions. In all cases, a good matching between the models and the measurements is obtained.
\end{abstract}

Index Terms-MOS capacitors, diffusive representation, charge trapping control, sliding control, ionizing radiation.

\section{INTRODUCTION}

Dielectric charge trapping is widely identified as a key factor affecting the reliability of many MOS-related devices [1]. The physical mechanisms responsible for such charge trapping are rather complex and, for a given device type, mainly dependent on the fabrication process, the temperature and the voltage applied [2]-[9]. Other factors, such as external disturbances (i.e. due to ionizing radiation) have also a remarkable influence on charge trapping.

The characterization of the charge trapping dynamics in MOS devices has been extensively studied [10]. Trap distributions are obtained from impedance spectra for different voltages in [11], [12]. Other works analyze the charging dynamics as the result of the application of voltage, or temperature, stress, while observing discharging currents or shifts in the threshold voltage. In these methods, the stress factor is instantaneously changed and changes in drain currents or threshold voltage shifts are monitored and fitted. Models with one or two time constants, as well as, more generally, stretched exponentials have been used to this purpose. In stretched exponentials the charge transient is characterized by the function $\exp \left(-(t / \tau)^{\beta}\right)$, with $\beta \in(0,1)$. This model has been linked to Gaussian distributions in the energy barriers found in charge trapping and detrapping [13]. However, in all these cases the analysis of the time evolution of systems under arbitrary excitation becomes difficult because it is necessary to take into account the distributed nature of the processes involved.

Besides, the diffusive nature of the charge trapping phenomena allows their behavioral characterization using state-space models based on Diffusive Representation. In recent times, Diffusive Representation tools have been successfully used for modeling and prediction purposes in several diffusion-based systems, such as thermal [14], [15] and electrical [16], [17] fractional systems. In this paper, we use Diffusive Representation since it allows to directly obtain models from experimental data, which can be very easily simulated and are compatible with physical processes including diffusion and dispersion in general.

On the other hand, charge trapping controls based on sigmadelta loops have been proposed recently for MOS capacitors in [18]. Under some limits, these controls allow to set and maintain a previously given amount of net dielectric charge, thus mitigating long-term reliability issues such as $\mathrm{C}-\mathrm{V}$ shifting or threshold voltage drifts. In these controls, the charge being continuously leaked out of the dielectric is compensated in average by applying an adequate sequence of bipolar voltages. The same type of sigma-delta strategies had been previously used to control the charge in MEMS [19][21]. In this case, Diffusive Representation and Sliding-Mode Control tools were used successfully to analyze and predict the dynamics of the trapped charge.

Furthermore, charge trapping generated by ionizing radiation in harsh environments such as space applications has been extensively studied. Changes in the threshold voltage of MOS transistors are among the most noticeable effects [22][26]. To mitigate this problem, extensive design and shielding strategies are routinely implemented. Besides, compensation of charge trapped in $\mathrm{SiO}_{2}$ MOS capacitors induced by gamma radiation using sigma-delta controls has been demonstrated recently in [27]. Ionizing radiation such as gamma and Xray radiation can be understood as an external disturbance in a sliding mode controller affecting the net charge in the dielectric [28].

Models based on continuous sliding mode controllers (SMCs) have been used to analyze the charge dynamics in MEMS [19] and to improve the response of wind sensors [29]. On the other hand, discrete-time SMCs proposed for applications such as position tracking control of linear motors [30], [31] allowed to achieve improved performance when compared to continuous SMCs. In addition, non-singular terminal sliding mode (NTSM) control, combined with finitetime observers [32] and backpropagating constraints [33] has been implemented for accurate tracking of systems with unknown dynamics affected by external disturbances.

According to the above, the objective of this paper is to introduce a new behavioral model, based on Diffusive Representation, to characterize the dynamics of the charge trapped in the dielectric of MOS capacitors. This model is an extension of the one used for MEMS in [20], [21]. The derivation of the model for an $\mathrm{Al}_{2} \mathrm{O}_{3}$ MOS capacitor and its effectiveness to reproduce and predict the evolution of the net trapped charge when the device is under open-loop excitations are explained and investigated in Section II. A 
second objective is to extend the model to the case in which the charge control from [18] is applied to the capacitor. To this effect, the behavior of the device under control is predicted using a specific analytical model, derived from the SlidingMode Control theory, in Section III. Experimental results have been obtained for MOS capacitors under sigma-delta control of charge, with and without ionizing radiation (X-rays). These results, shown and discussed in Section IV, demonstrate a good matching between model predictions and experimental data. It is also shown that, in accordance with model predictions, the trapped charge induced by radiation is not removed but can be successfully compensated by the control loop.

\section{DifFusive REPRESENTATION}

As mentioned above, in this work Diffusive Representation (DR) is used to model the dynamics of the charge trapped in the dielectric of MOS capacitors. This section successively describes the derivation of the DR model, the fabrication of the capacitors and, finally, the validation of the model with experimental data.

\section{A. Building the DR model}

Given a non-rational transfer function, $H(p)$, associated with a convolution causal operator denoted by $H\left(\partial_{t}\right)$, the diffusive realization of this operator is expressed by the following input $(u)$ - output $(y)$ state space realization of $u \longmapsto y=H\left(\partial_{t}\right) u=h * u$ of the form [16], [34]:

$$
\begin{aligned}
\dot{\psi}(\xi, t) & =-\xi \psi(\xi, t)+u(t), \quad \psi(\xi, 0)=0 \\
y(t) & =\int_{0}^{\infty} \eta(\xi) \psi(\xi, t) d \xi
\end{aligned}
$$

where $\xi \in \mathbb{R}$ is frequency and $\psi(\xi, t)$ the diffusive representation of the input [16]. The weight distribution of $\psi(\xi, t)$ in the output, $\eta(\xi)$, is the diffusive symbol of the operator.

The DR model used in this work is obtained by finite order approximation of the operator $H\left(\partial_{t}\right)$, with the continuous variable $\xi$ discretized into $\left\{\xi_{k}\right\}_{1 \leq k \leq N}$, where $N$ is the order of the model. This approach allows working with experimental data, which consist on periodical samples of the output (a variable related to the variation of the trapped charge), when specific time-varying input functions are applied to the device. These input functions, described later in this section, are sequences of voltage waveforms that imply switching between a reduced number of $M$ voltage levels. According to this, let us consider the input function $v(t): V \longrightarrow R$, of the form,

$$
v(t)=\sum_{i=1}^{M} V_{i} 1_{S_{i}}(t)
$$

where $1_{S_{i}}(t)$ is an indicator function, defined as,

$$
1_{S_{i}}(t)= \begin{cases}1, & v(t)=V_{i} \\ 0, & v(t) \neq V_{i}\end{cases}
$$

and where $\left\{S_{i}\right\}$ is a sequence of disjoint measurable sets. Now, the charge trapped is modelled as a time-varying linear system. The time evolution of such system can be described with the following set of $M \times N$ equations,

$$
\dot{\psi_{k}^{i}}(t)=-\xi_{k} \psi_{k}^{i}(t)+1_{S_{i}}(t), \quad i=1 \cdots M
$$

where $\left\{\xi_{k}\right\}_{1 \leq k \leq N}$ is the frequency mesh [20].

The state vector is $\psi(t)=\left(\psi^{1}(t) \psi^{2}(t) \ldots \psi^{M}(t)\right)^{T} \in$ $\mathbb{R}^{M}$, where $\psi^{i}(t)=\left(\psi_{1}^{i}(t) \psi_{2}^{i}(t) \ldots \psi_{N}^{i}(t)\right) \in \mathbb{R}^{N}$. This state vector is always continuous during the switching between voltages.

The output of the system can be expressed as,

$$
y(t)=\sum_{i=1}^{M} \sum_{k=1}^{N} \eta_{k}^{i} \psi_{k}^{i}(t)
$$

where $\eta_{k}^{i}$ is the diffusive symbol related to the frequency $\xi_{k}$ and the voltage $V_{i}$. The set of diffusive symbols related to each voltage $V_{i}$ is defined as $\eta^{i}=\left(\eta_{1}^{i} \eta_{2}^{i} \ldots \eta_{N}^{i}\right) \in \mathbb{R}^{N}$.

Since the device could be in an arbitrary charge status at the beginning of an experiment (i.e at $t=t_{0}$ ), adding an exponential decay term with coefficients $a_{k}$ allows including such 'initial condition' in the model. In this case, the output becomes:

$$
y(t)=\sum_{i=1}^{M} \sum_{k=1}^{N} \eta_{k}^{i} \psi_{k}^{i}(t)+\sum_{k=1}^{N} a_{k} e^{-\xi_{k}\left(t-t_{o}\right)}
$$

The diffusive symbols $\eta_{k}^{i}$ and the coefficients $a_{k}$ are obtained by fitting the output corresponding to the finite order approximation of the operator with experimental data. Here, the diffusive symbols obtained by pseudo-inverting $H(p)$ provide a close approximation to the operator.

The accuracy of this model depends on the distribution of the frequency mesh $\left\{\xi_{k}\right\}_{1 \leq k \leq N}$, which in our case is chosen to be geometrically spaced in the band of interest. The minimum and maximum frequencies depend respectively on the total duration of the measurements, $T$ (then $\xi_{\min }=2 \pi / T$ ), and on the sampling period, $T_{S}$ (then $\xi_{\max }=2 \pi / T_{S}$ ).

Finally, let us note that in our model the output of the system is the voltage shift of the $\mathrm{C}-\mathrm{V}$ characteristic of the device. The voltage shift is an indirect inference to the changes in the net charge trapped in the dielectric. This is compatible with the observations in [27], which indicated that charge injection changed when the net charge levels around which they were studied were far apart one of the other. In practice, this means that constant multi-exponential charge models are valid for MOS capacitors, so long as the total displacement of the net charge is not significantly changed.

\section{B. MOS capacitors and characterization waveforms}

The capacitors used in this work are all from the same fabrication batch, with same $1 \mathrm{~mm}^{2}$ area. They have been fabricated in our clean-room facilities from a p-type c-Si $<100>$ wafer of $280 \mu \mathrm{m}$ thick and $2.5 \pm 0.5 \Omega \mathrm{cm}$ resistivity. The process starts with RCA cleaning, followed by the deposition of a $20 \mathrm{~nm}$ thick $\mathrm{Al}_{2} \mathrm{O}_{3}$ layer on both sides of the wafer. Thermal atomic layer deposition at $200^{\circ} \mathrm{C}$ is used to this purpose. The front side of the wafer is covered with photoresist and then the $\mathrm{Al}_{2} \mathrm{O}_{3}$ layer on the rear side is etched. A $700 \mathrm{~nm}$ thick $\mathrm{Al}$ layer 
a)

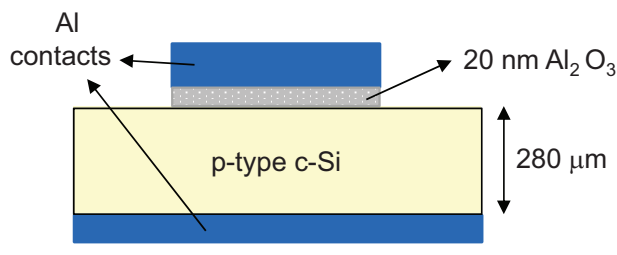

b)

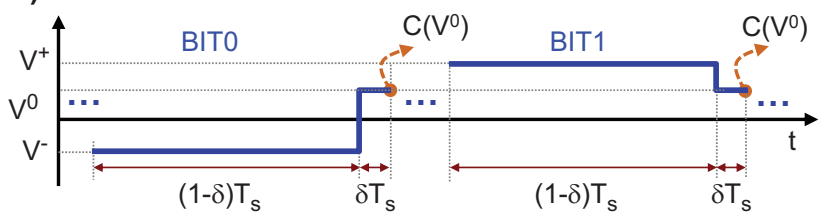

Fig. 1: a) Vertical schematic of the MOS capacitors. b) Voltage waveforms BIT0 and BIT1 applied to the capacitors. In the devices used, voltages $V^{+}$and $V^{-}$produce opposite C-V shifts, related to charge changes of different sign. To sense such shifts, a capacitance measurement at the constant reference voltage $V^{0}$ is performed at the end of each waveform.

is evaporated on top of the $\mathrm{Al}_{2} \mathrm{O}_{3}$. Next, photolithography is used to pattern the capacitors and the unwanted $\mathrm{Al}$ and $\mathrm{Al}_{2} \mathrm{O}_{3}$ are removed with isopropyl and phosphoric acid solution. Then the rear side of the wafer is covered with $\mathrm{Al}$ evaporated by electron beam to form the back contact. Finally, an annealing step at $350^{\circ} \mathrm{C}$ in $\mathrm{N}_{2}$ is performed. A vertical cross-section of the devices is depicted in Fig. 1(a).

On the other hand, two voltage waveforms, BIT0 and BIT1, have been used to characterize the dynamics of the charge trapped in the dielectric. These waveforms, originally designed for charge sensing and control in $\mathrm{SiO}_{2}$ devices [18], are described in Fig. 1(b). As it can be observed, during BIT0, a negative voltage $V^{-}$is applied to the device for a time $(1-\delta) T_{S}$, then a test voltage $V^{0}$ is applied for a short time $\delta T_{S}$, being $T_{S}$ the total duration of the waveform and $0<\delta<1$. BIT1 has the same shape, but with with positive voltage $V^{+}$applied instead of $V^{-}$. The capacitance of the device at the test voltage, $C\left(V^{0}\right)$, is measured at times $n T_{S}$.

As already seen in [18], for the $\mathrm{Al}_{2} \mathrm{O}_{3}$ devices and the voltage ranges used in this work, the application of either a BIT0 or a BIT1 produces opposite-horizontal shifting of the C-V characteristic. Since no other effects (i.e. C-V stretchingout) have been observed, this voltage shift can be related to variations of the net charge trapped in the $\mathrm{Al}_{2} \mathrm{O}_{3}$ layer. Additionally, the voltage shift can be easily monitored from the $\left.C\left(V^{0}\right)\right|_{n}$ measurements.

\section{Obtention and validation of the DR model}

A capacitor has been characterized through an experiment in which an open-loop sequence of BIT0s and BIT1s is the input data applied to the device. Concretely, a Pseudo Random Binary Sequence (PRBS) is used to this purpose. The PRBS is a random sequence of macro-bits, each one composed of 30 identical BIT0 (or BIT1) waveforms. The BITx parameters chosen are $V^{+}=V^{0}=1.5 \mathrm{~V}, V^{-}=-3 \mathrm{~V}, \delta=1 / 3$ and $T_{S}=420 \mathrm{~ms}$.
The voltage levels have been chosen to simplify the process of obtaining the DR model. Since the experiment length is 21 hours, the frequency ranges approximately from $f_{\text {min }}=8.5 \mu \mathrm{Hz}$ to $f_{\max }=0.1 \mathrm{~Hz}$, with values spaced geometrically.

The experiment has been divided into two parts of equal duration. During the first half, the diffusive symbols are extracted from fittings of the experimental data. The DR model derived is then used for prediction purposes in the second half of the experiment. There, the DR model simulation is compared with the remaining experimental data. Let us remark here that the data obtained from the experiment is the evolution of the sampled device capacitance at a given voltage, $\left.C\left(V^{0}\right)\right|_{n}$. However, since we are interested in the charge control characteristics in terms of shifts of the C$\mathrm{V}$ curves, the capacitance variation has been converted into voltage shift variation. Let us also remark that the DR model describes the time evolution of the net charge in the device for given constant voltages. For a given voltage, this net charge will generally be the result of the injection of both positive and negative charge.

Fig. 2 shows the evolution of both the experimental voltage shift and the fitted data during the first part of the experiment. The order of the DR model chosen, $N=5$, is the minimum allowing good accuracy in the fittings. Let us note that further increasing $N$ does not significantly improve the accuracy, as shown in [35]. The diffusive symbols inferred from the fitting, associated with the two voltages used, are shown in Fig. 3. As discussed above, the dependence of the obtained symbols only on the operator but not on the input allows modeling the charge/voltage shift dynamics of the device for arbitrary input sequences. The difference when a positive or negative voltage is applied can be observed in the diffusive symbol, as well as a difference in the amplitudes due to the absolute voltage values. The non-convergence of the diffusive symbols observed at high frequencies is due to the lack of high frequency components.

The next objective is to validate the model through comparison of its predictions with the experimental data obtained taking the second half of the PRBS as input signal. This step is necessary to investigate the capability of the model to describe the charge dynamics when other arbitrary waveforms using the same voltages are applied. The result of this second half of the experiment is reported in Fig. 4, where very good match between prediction and experimental data is observed.

\section{Charge trapping CONTROL}

In this section, the charge dynamics of a MOS capacitor under sigma-delta control is analyzed using the tools of sliding mode controllers for an infinite sampling frequency approximation. A simplified analytical model, allowing to predict and analyze the sequence of control signals, is introduced and validated with experimental data.

\section{A. Sigma-delta control of dielectric charging}

The control method used, taken from [18], is aimed to provide sliding mode control of the net charge trapped in the dielectric of a MOS capacitor. An schematic of the control loop is depicted in Fig. 5. The dielectric is seen as a reservoir 


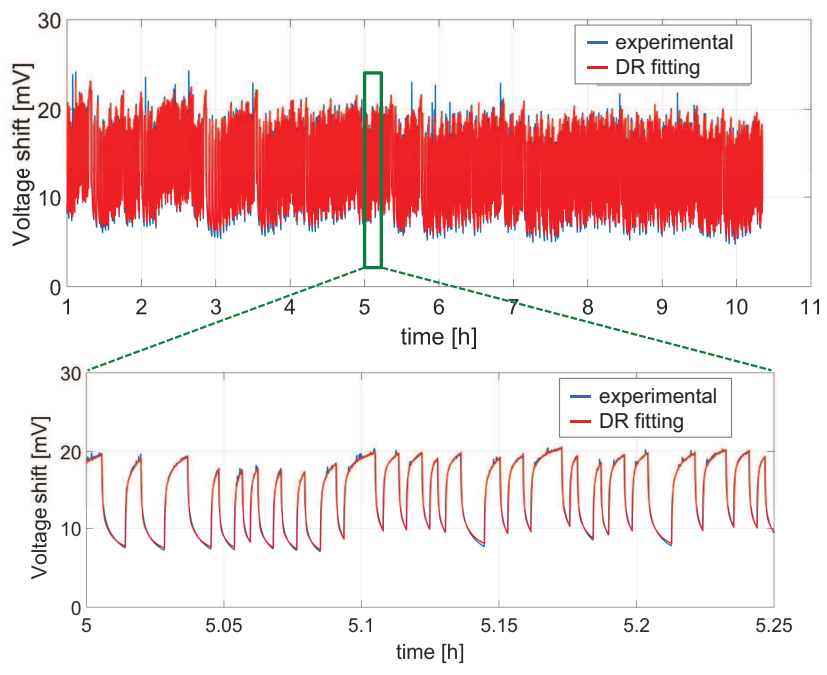

Fig. 2: Top: Evolution of the voltage shift of the C-V (in blue) and DR fitting (in red) for an experiment in which a PRBS was applied to the device for 10.5 hours. The root mean square error (RMSE) obtained from fitting is $0.69 \mathrm{mV}$. A 15 -minute zoom is shown at the bottom. The diffusive symbols obtained from the fitting are shown in Fig. 3.

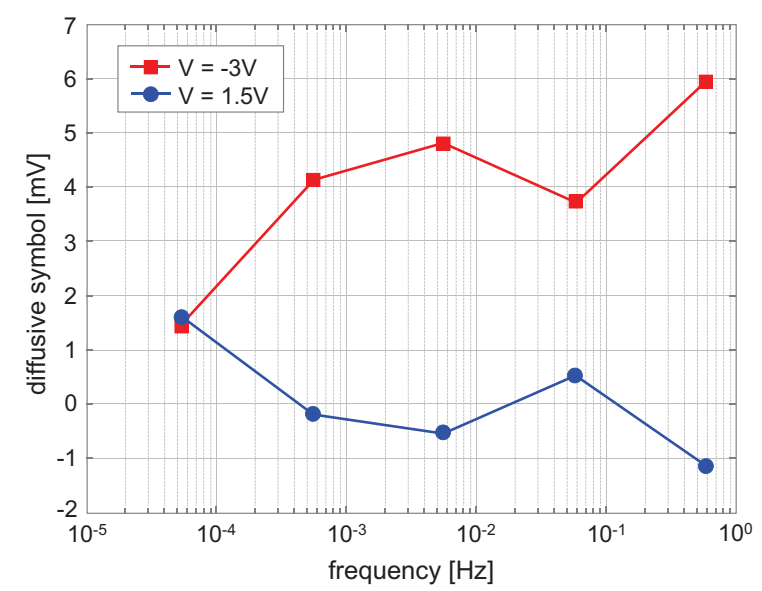

Fig. 3: 5-th order diffusive symbols of the voltage shift for the two voltages used in the BIT0 and BIT1 waveforms applied to the device: $V^{+}=+1.5 \mathrm{~V}$ and $V^{-}=-3 \mathrm{~V}$.

of trapped charge under three competing mechanisms: a) charge injected by the voltages applied, b) charge being leaked out, c) charge generated by radiation. The control signal, a sequence of voltage waveforms, tries in average to inject the necessary charge to keep the total dielectric charge constant.

As stated in the previous section, changes in the net charge produce horizontal (voltage) shifts of the $\mathrm{C}-\mathrm{V}$ curve of the device, detected from capacitance measurements $\left.C\left(V^{0}\right)\right|_{n}$ made at the sampling times $n T_{S}$. According to this, the control loop provides an intelligent actuation of the device, allowing to compensate charging effects and to keep $\left.C\left(V^{0}\right)\right|_{n}$ (or $\left.V_{s h}\right|_{n}$ ) at a target level $C_{t h}$ (or $V_{\text {target }}$ ). This is achieved by using the voltage waveforms BIT0 and BIT1, see Fig. 1.

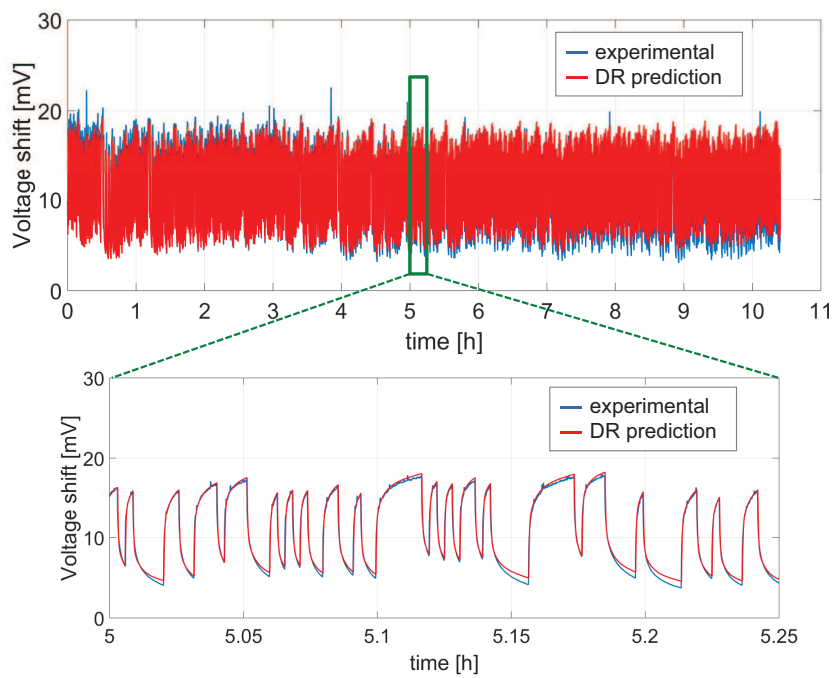

Fig. 4: Evolution of the voltage shift (in blue) versus the DR model prediction (in red) for an experiment in which a PRBS was applied to a MOS capacitor for 10.5 hours. The RMSE obtained from comparing the model prediction with the experimental data is $0.86 \mathrm{mV}$. This experiment followed that shown in Fig. 2. A 15-minute zoom is shown at the bottom.

In the devices used, applying BIT0s ( $V^{-}<0$ dominant) tends to increase the negative charge trapped in the dielectric and therefore to shift the $\mathrm{C}-\mathrm{V}$ to the right. On the other hand, applying BIT1s $\left(V^{+}>0\right.$ dominant) produces left $\mathrm{C}-\mathrm{V}$ shifting. Thus, by choosing between BIT0 and BIT1 at each sampling time, the sigma-delta loop tends to minimize the error signal $\left.C\left(V^{0}\right)\right|_{n}-C_{t h}\left(\right.$ or $\left.\left.V_{s h}\right|_{n}-V_{\text {target }}\right)$.

Additionally, the sequence of BITs applied (the bit stream generated by the loop, $b_{n}$ ) provides real-time information about the charge dynamics. Second order sigma-delta control has also been demonstrated for $\mathrm{SiO}_{2}$ capacitors in [36]. This second order strategy improves noise shaping and prevents the appearance of plateaus, which can hinder the effectiveness of the control in some cases.

Let us now model the charge control system. Firstly, waveforms BIT0 and BIT1 can be described as:

$$
\begin{aligned}
& v_{\text {bit } 0}(t)= \begin{cases}V_{1}, & t \in\left[0,(1-\delta) T_{S}\right) \\
V_{2}, & t \in\left[(1-\delta) T_{S}, T_{S}\right) \\
0, & t \notin\left[0, T_{S}\right)\end{cases} \\
& v_{\text {bit } 1}(t)= \begin{cases}V_{3}, & t \in\left[0,(1-\delta) T_{S}\right) \\
V_{2}, & t \in\left[(1-\delta) T_{S}, T_{S}\right) \\
0, & t \notin\left[0, T_{S}\right)\end{cases}
\end{aligned}
$$

where $V_{1}=V^{-}, V_{2}=V^{0}$ and $V_{3}=V^{+}$.

Taking into account the time-varying linear system described in the previous section, the actuation waveforms and 


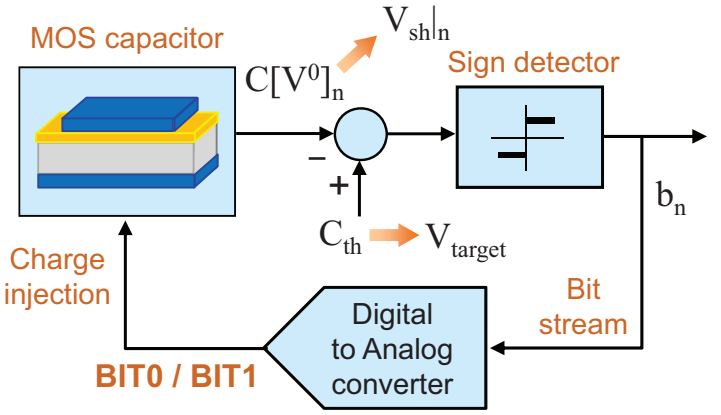

Fig. 5: First-order charge control loop [18]. The net charge trapped in the dielectric produces horizontal $\mathrm{C}-\mathrm{V}$ shifts, sensed through capacitance measurements performed at the reference voltage $V^{0}$. At each sampling time, the measured capacitance $\left.C\left(V^{0}\right)\right|_{n}$ is compared to a target value $C_{t h}$ (related to a target voltage shift $\left.V_{\text {target }}\right)$ and, depending on the result, either a BIT0 or a BIT1 waveform is applied to the device in the next sampling period.

the sigma-delta control loop, one can write:

$$
\begin{aligned}
v(t)= & \frac{1}{2} \sum_{n}\left(1+b_{n}\right) v_{\mathrm{bit} 1}\left(t-n T_{S}\right) \\
& +\left(1-b_{n}\right) v_{\mathrm{bit} 0}\left(t-n T_{S}\right) \\
\dot{\psi}_{k}^{i}(t)= & -\xi_{k} \psi_{k}^{i}(t)+1_{s_{i}}(t) \\
V_{s h}(t)= & \sum_{i=1}^{M} \sum_{k=1}^{N} \eta_{k}^{i} \psi_{k}^{i}(t) \\
b_{n}= & \operatorname{sgn}\left(V_{s h}\left(n T_{S}\right)-V_{\text {target }}\right)
\end{aligned}
$$

where $1_{S_{i}}(t)$ was defined in (2), $b_{n}$ is the control bit stream, and $V_{s h}$ and $V_{\text {target }}$ are respectively the actual and the target values of the voltage shift.

\section{B. Deterministic switching: average system}

The voltage waveforms BIT0 and BIT1 present a two level hierarchy. The first level is voltage switching within each waveform and it provides an instantaneous amount of voltage shift at each waveform end. The second level is the switching between different waveforms, which depends on the instantaneous value of the net charge at the sampling times $n T_{S}$ and therefore on the sampled state vector, $\psi$.

Let us now assume the 'infinite sampling approximation', which in this case implies that the sampling period, $T_{S}$, is at least one order of magnitude below the shortest time constant in the affine models. In the limit $T_{S} \rightarrow 0$, an 'average system', where the continuous voltage switching within the bits is no longer present, can be considered. According to this, the time evolution of the state variables under the application of either BIT0 or BIT1 can be written as:

$$
\dot{\psi}_{k}^{i}(t)=-\xi_{k} \psi_{k}^{i}(t)+\alpha_{0}^{i} 1_{b_{0}(t)}+\alpha_{1}^{i} 1_{b_{1}(t)}
$$

where the function $1_{b_{x}}(t)$ indicates whether a BIT0 or a BIT1 is applied at time $t$ (then $\sum_{x} 1_{b_{x}}(t)=1$ ) and $\alpha_{x}^{i}$ is the percentage of time within BITx in which the voltage
$V_{i}, i \in[1, \ldots, M]$, is applied. This therefore means that $0 \leq \alpha_{x}^{i} \leq 1$ and $\sum_{i} \alpha_{x}^{i}=1$. To illustrate this, consider three voltage levels (M=3), $V_{1}, V_{2}$, and $V_{3}$, then the waveforms for BIT0 and BIT1 can be expressed as

$$
\begin{aligned}
& v_{\text {bit0 }}(t)=V_{1} 1_{\left[0,(1-\delta) T_{S}\right)}(t)+V_{2} 1_{\left[(1-\delta) T_{S}, T_{S}\right]}(t) \\
& v_{\text {bit1 }}(t)=V_{3} 1_{\left[0,(1-\delta) T_{S}\right)}(t)+V_{2} 1_{\left[(1-\delta) T_{S}, T_{S}\right]}(t)
\end{aligned}
$$

From this, we get $\left(\alpha_{0}^{1}, \alpha_{0}^{2}, \alpha_{0}^{3}\right)=(1-\delta, \delta, 0)$ for BIT0 and $\left(\alpha_{1}^{1}, \alpha_{1}^{2}, \alpha_{1}^{3}\right)=(0, \delta, 1-\delta)$ for BIT1, respectively.

The dynamics of this system under the sigma-delta control can be then described as:

$$
\dot{\psi}_{k}^{i}(t)=-\xi_{k} \psi_{k}^{i}(t)+\alpha_{0}^{i} \frac{1-\operatorname{sgn}(\sigma)}{2}+\alpha_{1}^{i} \frac{1+\operatorname{sgn}(\sigma)}{2}
$$

where $\sigma=V_{\text {sh }}(t)-V_{\text {target }}$.

Finally, equations (15) can be written as:

$$
\dot{\psi}_{k}^{i}(t)= \begin{cases}-\xi_{k} \psi_{k}^{i}(t)+\alpha_{0}^{i}, & \sigma<0, \\ -\xi_{k} \psi_{k}^{i}(t)+\alpha_{1}^{i}, & \sigma>0,\end{cases}
$$

Further details on the implementation of the control system described by Fig. 5 and equations (15) and (16) are provided in section IV.C below.

\section{Sliding mode analysis}

The set of equations (16) can be seen as the description of a particular case of sliding mode controller, in which the sliding function is $\sigma=V_{s h}(t)-V_{\text {target }}$ and where the time dynamics of two average systems depend on whether the state vector is on one side $(\sigma<0)$ or the other $(\sigma>0)$ of the control surface $\sigma=0$. This result is very similar to that obtained for charge control in MEMS [19] and is in perfect accordance with the relationship between sliding mode control and sigma-delta modulation explained in [37].

In general, the sliding mode control can be understood as consisting on two successive phases: (a) reaching phase, where the control applies a constant sequence of actuation waveforms (i.e only BIT0 or BIT1) to make the average system reach the sliding surface $(\sigma=0)$ from an arbitrary initial position, and (b) sliding phase, during which the state of the system moves along the sliding surface. The time evolution of the state variables is determined by the equivalent control necessary to maintain the system within the control surface.

Assuming that the system reaches the control surface $\sigma=0$ and that an attractive sliding domain exists with the control surface, the ideal sliding dynamics on $\sigma=0$ is described by Filippov's regularization is a convex combination of the vector fields applied in both sides of the control surface:

$$
\dot{\psi_{k}^{i}}(t)=-\xi_{k} \psi_{k}^{i}(t)+\lambda \alpha_{1}^{i}+(1-\lambda) \alpha_{0}^{i}
$$

where $0 \leq \lambda \leq 1$ must coincide with the average values of the bit stream (properly windowed by a low pass filter) and must ensure that $\dot{\sigma}$ must be zero i.e., the system slides on the control surface. It is important to note that, in fact, the average bit stream $\lambda$ depends on the state vector $\psi$. However, to simplify the notation we will not explicitly indicate this dependence in the next equations. 
Taking into account the expression of the voltage shift given in (11), the condition $\dot{\sigma}=0$ leads to:

$$
\dot{V}_{s h}(t)=\sum_{i=1}^{M} \sum_{k=1}^{N} \eta_{k}^{i}\left(-\xi_{k} \psi_{k}^{i}(t)+\lambda \alpha_{1}^{i}+(1-\lambda) \alpha_{0}^{i}\right)=0
$$

The solution of the above equation is $\lambda$, the control equivalent which keeps the system on the control surface:

$$
\lambda=\frac{\sum_{i} \sum_{k} \eta_{k}^{i} \xi_{k} \psi_{k}^{i}(t)-\sum_{i} \sum_{k} \eta_{k}^{i} \alpha_{0}^{i}}{\sum_{i} \sum_{k} \eta_{k}^{i}\left(\alpha_{1}^{i}-\alpha_{0}^{i}\right)}
$$

Now, expressions (17) and (19) together provide the nonlinear equation evaluating the time response of the system after reaching the sliding surface, $\sigma=0$.

\section{EXPERIMENTAL RESULTS AND DISCUSSION}

\section{A. Experiment 1: Charge trapping control}

The first objective of this experiment is to investigate the effectiveness of the DR model when charge control is applied to a MOS capacitor. Another objective is to verify that the control system behaves as a sliding controller and, therefore, it can be interpreted and analyzed using the analytical model developed in the previous section. According to this, sigmadelta control is applied to an $\mathrm{Al}_{2} \mathrm{O}_{3}$ capacitor to set a constant target voltage shift for 25 hours. The BITx parameters used are: $V^{+}=V^{0}=1.5 \mathrm{~V}, V^{-}=-3 \mathrm{~V}, T_{S}=350 \mathrm{~ms}$ and $\delta=1 / 3$. The target voltage shift is $V_{\text {target }}=7.8 \mathrm{mV}$, which for the device chosen corresponds to a target capacitance $C_{t h}=0.6 \mathrm{nF}$ at $V^{0}=1.5 \mathrm{~V}$. As in previous experiments, a precision LCR Meter generates the BITx waveforms and measures the capacitance of the device $C\left(V^{0}\right)$ at times $n T_{S}$. The decisions of the control algorithm and the data processing are implemented with a simple computer program.

Fig. 6 provides a comparison between the experimental results obtained (in blue), and

- the discrete time simulation of the 5-th order DR model obtained in the characterization process (in red), and

- the analytical expressions (17) and (19) obtained from the Sliding Mode analysis of the dynamics of the system when assuming an infinite sampling approximation (in green).

In Fig. 6(a) it is seen that, after a short initial transient of about 15-20 minutes, the target value is reached. The target value is then maintained successfully during the entire experiment by applying an adequate sequence of voltage waveforms. This long-term phase corresponds to the sliding phase in SMC and it can be seen as a straight (green) line in Fig. 6(a). On the other hand, Fig. 6(b) shows the slow time evolution of the bit stream during the sliding phase. Similar behavior has been observed in other devices, in which this type of control has been implemented [20], [35]. Finally, taking into account the similarity between the three curves in Fig. 6(b), the infinite sampling approximation is very suitable to understand how the control works.

Furthermore, the evolution of the bit stream provides additional information about the behavior of the system. In the initial transient only BIT1s are applied to the device. This means that injection of positive charge is necessary to
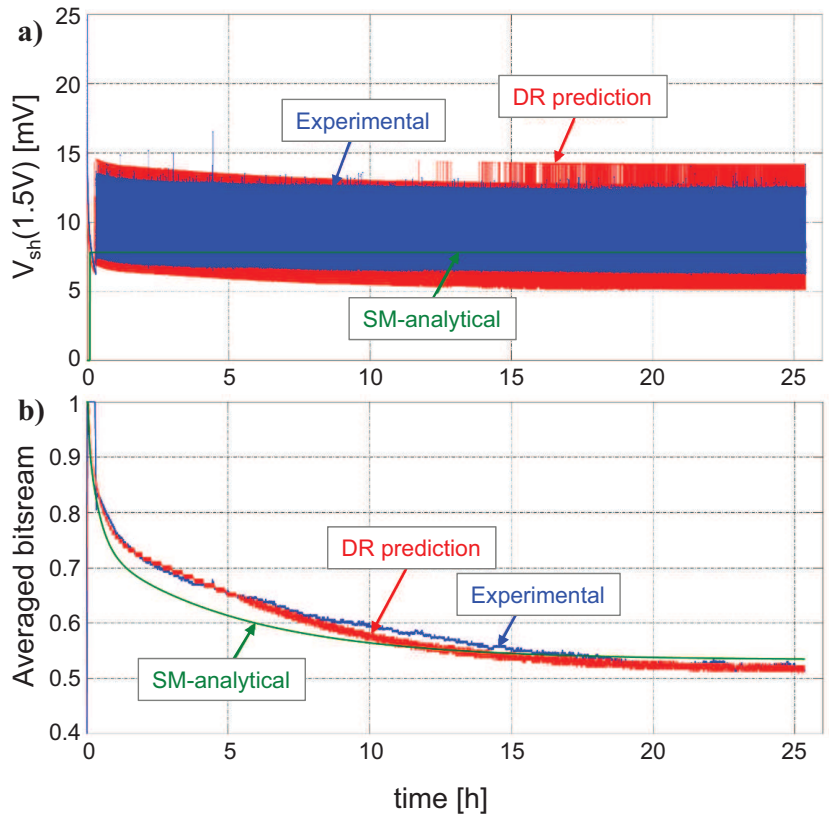

Fig. 6: Comparison of experimental data (in blue) with diffusive representation (in red) and sliding mode control (in black) predictions for a MOS capacitor in which charge control was applied for 25 hours. a) Evolution of the voltage shift of the $\mathrm{C}-\mathrm{V}$ curve. b) Evolution of the bit stream provided by the control loop.

compensate an initial amount of net negative dielectric charge. This produces left-shifting of the C-V until $V_{\text {target }}=7.8 \mathrm{mV}$ is obtained. From then on, control is achieved and therefore the bit stream becomes progressively stabilized. Note that the bit stream shows a very long transient, which means that by the end of the experiment the steady-state has not completely been reached.

Summarizing, as shown in Fig. 6(a), the DR model simulation demonstrates perfect agreement with experimental data, thus validating diffusive representation as a powerful prediction tool for this type of systems. On the other hand, the analytical model allows straight interpretation of the system behavior in terms of sliding mode control.

\section{B. Adding disturbances}

Let us now investigate the case in which the device, with or without control, is under an external disturbance such as ionizing radiation. As it will be shown experimentally in the next section, ionizing radiation creates additional charge trapping that should be added to the analysis of the previous sections.

According to this, let us suppose that the voltages applied to the device have an almost negligible effect on the dynamics of the charge generated by the disturbance. Under this consideration, the effect of this charge can be seen as an additional term in (17) and consequently as a shift of the C-V of value 
$\phi(t)$. Then, the 'effective' voltage shift becomes:

$$
V_{s h}(t)=\sum_{i=1}^{M} \sum_{k=1}^{N} \eta_{k}^{i} \psi_{k}^{i}(t)+\phi(t)
$$

and the new expression for $\lambda$ is:

$$
\lambda=\frac{\sum_{i} \sum_{k} \eta_{k}^{i} \xi_{k} \psi_{k}^{i}(t)-\sum_{i} \sum_{k} \eta_{k}^{i} \alpha_{0}^{i}-\dot{\phi}(t)}{\sum_{i} \sum_{k} \eta_{k}^{i}\left(\alpha_{1}^{i}-\alpha_{0}^{i}\right)}
$$

This result can be interpreted as follows: an external disturbance produces changes in both the control surface and the average bit stream that are necessary to keep the voltage shift (or the net charge) constant at the target value $V_{\text {target }}$.

This extended analytical model has been used to predict the effect of external disturbances in the control experiment shown in Fig. 6. To approach the charge generation due to ionizing radiation in a scenario similar to the experiment reported in the next section, the disturbance transients are divided into three steps: 1) no radiation (then $\phi=0$ ) during the first 2 hours, 2) radiation, modeled as constant charge generation rate ( $\phi=\beta t)$, for the next 8 hours, 3 ) no radiation (then $\phi$ constant) for the last 6 hours. This disturbance model is compatible with trapped charge being generated by radiation that is never removed from the dielectric. The bit streams necessary to keep the same target voltage shift for four different values of the generation ratio $\beta$ are shown in Fig. 7. It is seen that the increasing amounts of positive charge generated between $t=2$ and $\mathrm{t}=10$ hours are compensated by the control by increasing the number of BIT0s, which eventually increase the negative charge. When the disturbance episode ceases, the average bit streams always tend to stable-constant values. It is also seen that the charge generated by radiation is successfully compensated, but not removed.

The disturbance model developed helps to understand the influence of external disturbances on the closed loop control of the device. In particular, the model demonstrates the effect of ionization radiation dose rate on the amount of trapped charge generated (thus the amount of voltage shift) and the average bit streams needed to compensate it.

\section{Experiment 2: Charge control under ionizing irradiation}

This experiment investigates the effects of ionizing radiation on $\mathrm{Al}_{2} \mathrm{O}_{3}$ MOS capacitors under both open loop and charge control conditions. A specific objective is to check the goodness of the predictions of the sliding analytical model introduced when charging generated by the radiation occurs. To this effect, two MOS capacitors have been simultaneously irradiated with X-rays.

The experimental set-up is described in Fig. 8. The first capacitor, DUT1, is kept at constant voltage $V_{\text {bias }}=1 \mathrm{~V}$, and C$\mathrm{V}$ measurements are performed in 1 hour intervals during the experiment. The second device, DUT2, is under sigma-delta control to achieve and maintain a given target capacitance $C_{\mathrm{th}}$. Given the conditions of the experiment, a second-order loop is used to improve the reliability of the control, avoiding potential effects seen in first-order loops such as the appearance of plateaus [36]. In this case the capacitance of DUT2 at voltage $V^{0}$ is sampled periodically and compared with $C_{t h}$ to obtain

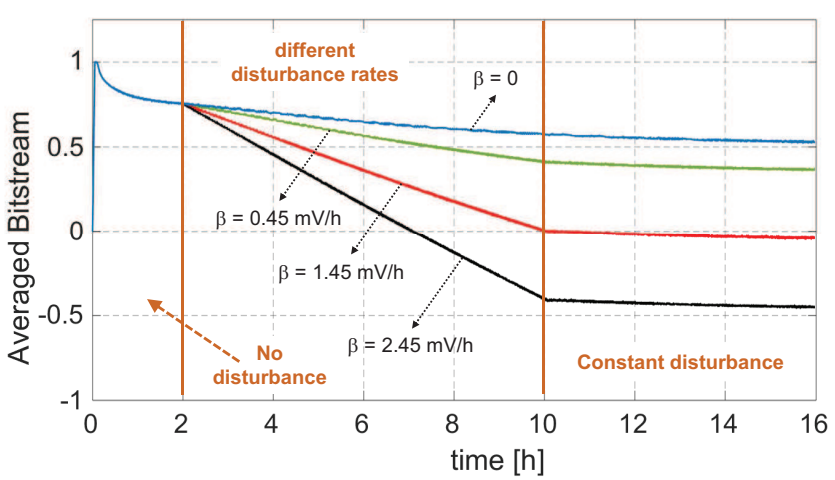

Fig. 7: Evolution of the bit stream provided by the control loop, as predicted by the sliding analytical model, when a set of disturbance functions, with different charge generation rates $(\beta)$, are applied for $8 \mathrm{~h}$ during the experiment reported in Fig. 6.

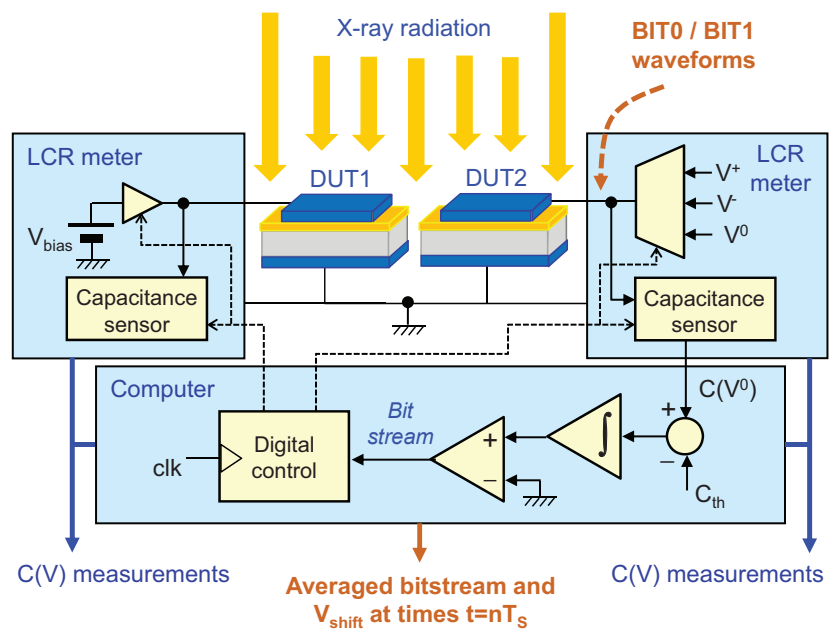

Fig. 8: Set-up used in experiment 2. Two devices, DUT1 and DUT2, are irradiated simultaneously, being DUT1 under constant voltage bias and DUT2 under second order charge control.

an error signal, which is then integrated and depending on the sign of the integral, either a BIT1 or BIT0 waveform is applied during the next sampling period.

The voltages used in BIT0 and BIT 1 are $V^{+}=5 \mathrm{~V}, V^{-}=-3 \mathrm{~V}$ and $V^{0}=1.5 \mathrm{~V}$, whereas the target capacitance is $C_{t h}=0.6 \mathrm{nF}$. The timing parameters are $T_{S}=350 \mathrm{~ms}$ and $\delta=1 / 3$, being $T_{S}$ smaller than the fastest time constant observed in the charging dynamics when these voltages are applied. Two precision LCR Meters generate the BITx waveforms and perform the capacitance measurements. Finally, let us note that DUT1 and DUT2 are pristine devices and therefore have comparable charge status, i.e. similar $\mathrm{C}-\mathrm{V}$, at the beginning of the experiment.

The experiment starts with no radiation for 90 minutes, followed by X-ray irradiation for 9 hours (at a constant dose rate of $10 \mathrm{~Gy} / \mathrm{h}$ ), and an 8-hour final stage with no radiation. Fig. 9(a) shows the evolution of the C-V of DUT1, which is 


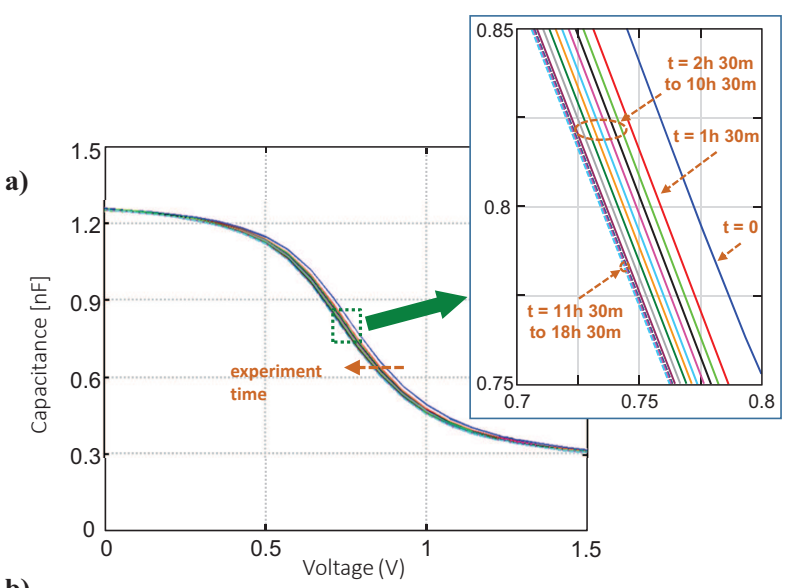

b)

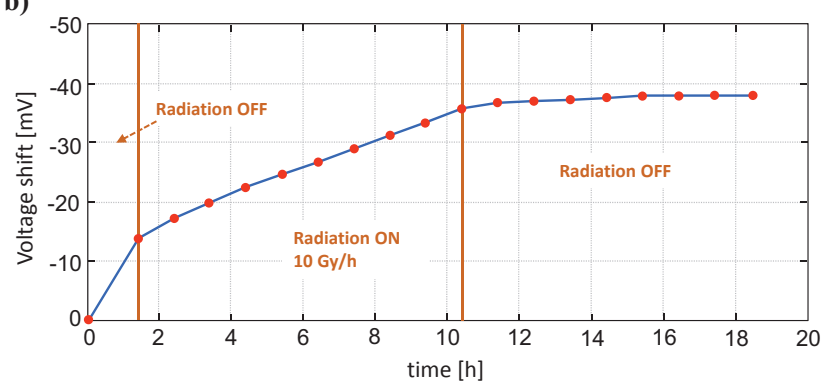

Fig. 9: Results obtained with DUT1 in experiment 2. The device was under constant $V_{\text {bias }}=1 \mathrm{~V}$. a) $\mathrm{C}-\mathrm{V}$ curves measured periodically during the experiment. The inset shows a closer view of the transition region of the C-Vs. b) Evolution of the voltage shift of the $\mathrm{C}-\mathrm{Vs}$ during the experiment. Noticeable left-shifting due to positive charge induced by the radiation is observed.

under constant $1 \mathrm{~V}$ bias. The C-Vs exhibit noticeable rigid leftshifting during the irradiation stage. Since other effects such as stretch-out are not observed, it can be concluded that the shifts are produced by increasing amounts of positive net charge trapped in the dielectric during irradiation [38]. Additionally, a constant shift rate of $0.25 \mathrm{mV} / \mathrm{Gy}$ during the irradiation phase is observed in Fig. 9(b). It is also seen that the device starts getting stabilized once radiation is turned off, and that the charge generated in the radiation phase remains once radiation ceases. This indicates that the charge accumulated by radiation is not removed.

On the other hand, the top graph in Fig. 10 shows the evolution of the capacitance at $V^{0}=1.5 \mathrm{~V}$ of DUT2, which is under charge control. During the initial stage (radiation off) the control acts until (at $\mathrm{t}=30$ minutes) the target capacitance $C_{t h}=0.6 \mathrm{nF}$ is achieved. Once this value is reached, it is successfully maintained during the rest of the experiment, irrespective of whether the radiation is on or off.

The bottom graph in Fig. 10 shows the evolution of the bit stream provided by the control loop. It illustrates how the control works; initially only BIT1s are applied, accumulating positive charge in the dielectric and moving the $\mathrm{C}-\mathrm{V}$ to the left to reach $C_{t h}$. During the irradiation stage (from t=90 min to $\mathrm{t}=10 \mathrm{~h} 15 \mathrm{~min}$ ), the control increases the number of BIT0s,

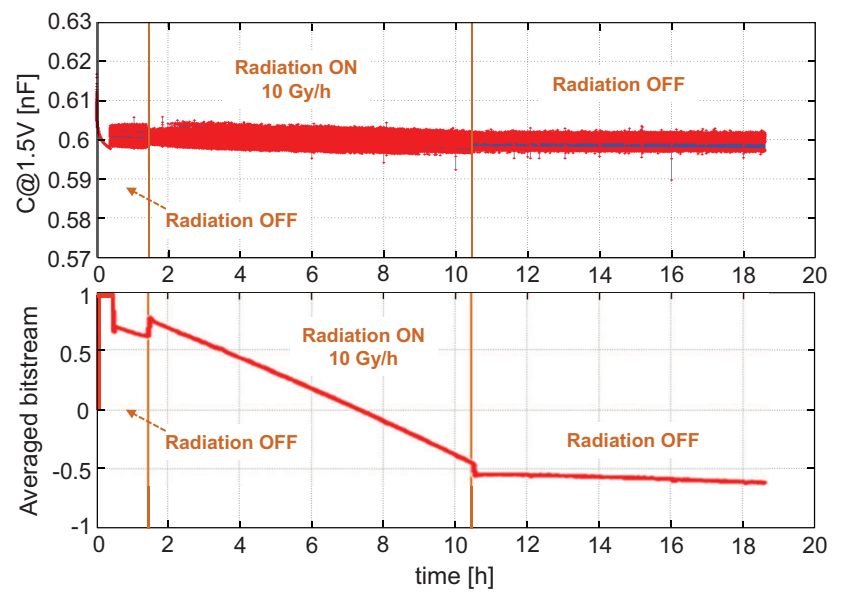

Fig. 10: Evolution of the capacitance at $V^{0}=1.5 \mathrm{~V}$ (top) and of the control bit stream (bottom) of DUT2 during experiment 2. The device was under second order charge control with target capacitance $C_{t h}=0.6 \mathrm{nF}$.

thus injecting more negative charge that compensates the accumulation of positive charge produced by the radiation. Once irradiation stops, the accumulated-remaining charge leads the bit stream to stabilize. A straight relationship between the voltage shifts of Fig. 9 and the control bit stream of Fig. 10 can also be observed. Additionally, Fig. 11 shows three C-V curves of DUT2, measured during the experiment. As expected, once the target value is reached (at $\mathrm{t}=45 \mathrm{~m}$ ) the $\mathrm{C}-\mathrm{Vs}$ are hardly distinguishable.

Finally, let us note that the experimental bit stream shown in Fig. 10 matches with the predictions of the sliding control model summarized in Fig. 7. As in experiment 1, this allows explaining the experimental results obtained in terms of a sliding mode controller: once the system reaches the target capacitance, or the control surface, the applied bit stream keeps the capacitance constant. Exposure to ionizing radiation generates an additional term, as in (20), that works as a mismatched disturbance, since the average bit stream is continuous. The changes in the dynamics generated by this disturbance clearly follow the predictions obtained in Section IV.B.

\section{CONCLUSION}

Diffusive representation has been used to characterize the dynamics of charge trapping in MOS capacitors, seen as timevarying diffusive systems in which time variability comes from the voltages applied to the devices. Experimental corroboration has been obtained. The obtained time-varying model has very good agreement with the experimental data obtained in both open and closed loop actuation strategies. Moreover, the statespace models are very well suited to describe the behavior of such diffusive systems under non-trivial controls, as sliding mode controllers.

Experiments involving ionizing radiation in MOS capacitors under charge control have also been presented. The results indicate that the trapped charge induced by radiation is not removed but successfully compensated by the control loop. 


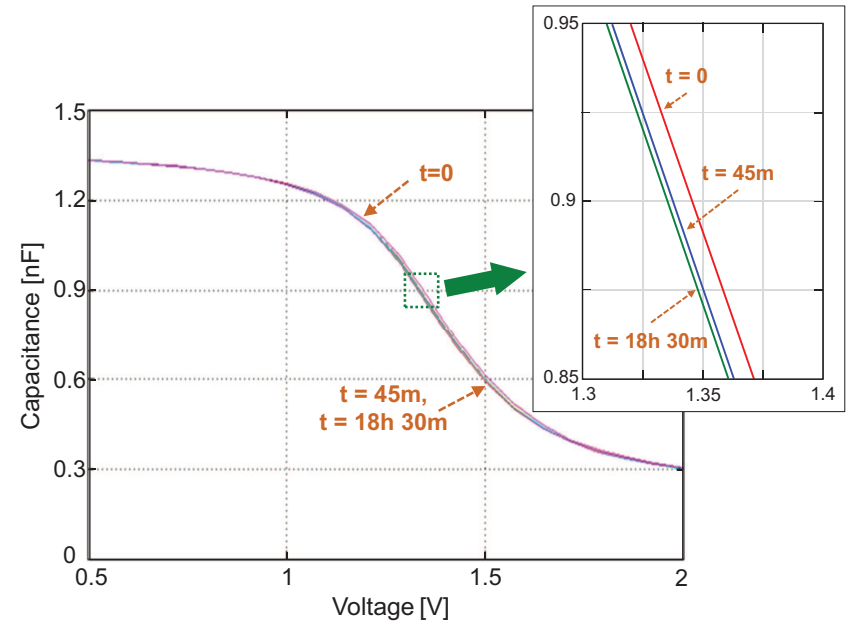

Fig. 11: C-V curves of DUT2 measured at three different times of the experiment reported in Fig. 10. The inset shows a closer view of the transition region of the C-Vs.

Applying the theory of sliding mode controllers, the changes observed in the control bit streams due to radiation are compatible with the effect of mismatched external disturbances on the system dynamics.

\section{REFERENCES}

[1] M. Razeghi, Fundamentals of Solid State Engineering. Second Edition. Springer, 2006.

[2] S. Zafar, A. Callegari, E. Gusev, and M. V. Fischetti "Charge trapping related threshold voltage instabilities in high permittivity gate dielectric stacks," Journal of Applied Physics, vol. 93, pp. 9298-9303, 2003.

[3] A. Gehring and S. Selberherr, "Modeling of tunneling current and gate dielectric reliability for non volatile memory devices," IEEE Trans. on Device and Materials Reliability, vol. 4, no. 3, pp. 306-319, 2004.

[4] V. Huard, M. Denais and C. Parthasarathy, "NBTI degradation: From physical mechanisms to modeling," Microelectronics Reliability, vol. 46, no. 1 , pp. 1-23, 2006.

[5] T. Grasser, B. Kaczer, W. Goes, T. Aichinger, P. Hehenberger, and M. Nelhiebel, "Understanding negative bias temperature instability in the context of hole trapping," Microelectronic Engineering, vol. 86, no. 7-9, pp. 1876-1882, 2009.

[6] T. Grasser "Stochastic charge trapping in oxides: from random telegraph noise to bias temperature instabilities," Microelectron. Reliab, vol. 52, pp. 39-70, 2012.

[7] S. Mahapatra, N. Goel, S. Desai, S. Gupta, B. Jose, S. Mukhopadhyay, K. Joshi, A. Jain, A. E. Islam and M. A. Alam "A Comparative Study of Different Physics-Based NBTI Models," IEEE Trans. on Electron Devices, vol. 60, no.3, pp. 901-916, 2013.

[8] Z. Y. Tung and D. S. Ang, "Impact of Voltage-Accelerated Stress on Hole Trapping at Operating Condition," IEEE Electron Device Letters, vol. 37, no. 5, pp. 644-647, 2016.

[9] S. H. Yoon, C. Y. Chang, D. H. Ahn, M. Takenaka and S. Takagi, "Interface state generation of A12O3/InGaAs MOS structures by electrical stress," Microelectronics Engineering, vol. 178, pp. 313-317, 2017.

[10] J.H. Stathis and S. Zafar, "The negative bias temperature instability in MOS devices: A review", Microelectronics Reliab., vol. 46, no. 2-4, pp. 270-276, 2006.

[11] Y. Yuan, L. Wang, B. Yu, B. Shin, J. Ahn, P.C. McIntyre, P.M. Asbeck, M.J.W. Rodwell and Y. Taur, "A Distributed Model for Border Traps in A12O3 InGaAs MOS Devices", IEEE Electron Device Letters, vol. 32, no. 4, pp. 485-487, 2011.

[12] Y. Yuan, B. Yu, J. Ahn, P.C. McIntyre, P.M. Asbeck, M.J.W. Rodwell and Y. Taur, "A Distributed Bulk-Oxide Trap Model for Al2O3 InGaAs MOS Devices", IEEE Trans. on Electron Devices, vol. 59, no. 8, pp. 2100-2106, 2012.

[13] O. Endholm and C. Blomberg, "Stretched exponentials and barrier distributions", Chemical Physics, vol. 252, no. 1-2, pp. 221-225, 2000.
[14] B. Allard, X. Jorda, P. Bidan, A. Rumeau, H. Morel, X. Perpina, M. Vellvehi and S. M'Rad, "Reduced-Order Thermal Behavioral Model Based on Diffusive Representation," IEEE Trans. Power Electron., vol. 24, no. 12, pp. 2833-2846, 2009.

[15] S. M'Rad, B. Allard, H. Morel, A. Ammous and N. Masmoudi "Analytic thermal modeling of power electronic components: The Diffusive Representation," 4th Int. Conf. on Integrated Power Syst., pp. 1-6, 2006.

[16] L. Laudebat, P. Bidan and G. Montseny, "Modeling and Optimal Identification of Pseudo-differential Electrical Dynamics by means of Diffusive Representation-Part I: Modeling," IEEE Trans. Circ. and Syst. I: Regular Papers, vol. 51, no. 9, pp. 1801-1813, 2014.

[17] C. Restrepo, G. Garcia, J. Calvente, R. Giral and L. Martinez-Salamero, "Static and Dynamic Current-Voltage Modeling of a Proton Exchange Membrane Fuel Cell Using an Input-Output Diffusive Approach," IEEE Trans. Ind. Electron., vol. 63, no. 2, pp. 1003-1015, 2016.

[18] M. Dominguez-Pumar, C. Bheesayagari, S. Gorreta, G. Lopez, I. Martin, E. Blokhina and J. Pons-Nin, "Charge Trapping Control in MOS Capacitors," IEEE Trans. Ind. Electron., vol. 64, no. 4, pp. 3023-3029, 2017.

[19] M. Domínguez-Pumar, S. Gorreta and J. Pons-Nin, "Sliding-Mode Analysis of the Dynamics of Sigma-Delta Controls of Dielectric Charging," IEEE Trans. Ind. Electron., vol. 63, no. 4, pp. 2320-2329, 2016.

[20] M.T. Atienza, S. Gorreta, J. Pons-Nin and M. Dominguez-Pumar, "Characterization of dielectric charging in MEMS using Diffusive Representation," Ind. Electron, vol. 64, no. 2, pp. 1529-1533, 2017.

[21] M.T. Atienza, L. Kowalski, S. Gorreta, J. Pons-Nin, V. Jiménez, and M. Dominguez-Pumar, "Model identification of Time-Varying Diffusive Systems," IEEE Int. Symp. on Circuits and Systems, pp. 1-5, 2018.

[22] D.M. Fleetwood, "Total Ionizing Dose effects in MOS and Low-DoseRate-Sensitive Linear-Bipolar Devices," IEEE Trans. Nucl. Sci., vol. 60, no. 3, pp. 1706-1730, June 2013.

[23] J.R. Schwank, M.R. Shaneyfelt, D.M. Fleetwood, J.A. Felix, P.E. Dodd, P. Paillet and V. Ferlet-Cavrois, "Radiation Effects in MOS Oxides," IEEE Trans. Nucl. Sci., vol. 55, no. 4, pp. 1833-1853, September 2008.

[24] X.J. Zhou, D.M. Fleetwood, J.A. Felix, E.P. Gusevand and C. D'Emic, "Bias-Temperature Instabilities and Radiation Effects in MOS Devices," IEEE Trans. Nucl. Sci., vol. 52, no. 6, pp. 2231-2238, February 2006.

[25] H.L. Hughes and J.M. Benedetto, "Radiation Effects and Hardening of MOS Technology: Devices and Circuits," IEEE Trans. Nucl. Sci., vol. 50, no. 3, pp. 500-521, July 2003.

[26] C. Claeys and E. Simoen, Radiation Effects in Advanced Semiconductor Materials and Devices, Springer Science Business Media, 2013.

[27] M. Dominguez-Pumar, C. Bheesayagari, S. Gorreta, G. Lopez and J. Pons-Nin, "Closed-loop compensation of charge trapping induced by ionizing radiation in MOS capacitors," IEEE Trans. Ind. Electron, vol. 65, no. 3, pp. 2518-2524, 2018.

[28] M. Domínguez-Pumar and S. Gorreta and J. Pons-Nin and F. GómezRodríguez and D.M. González-Castaño, "Charge induced by ionizing radiation understood as a disturbance in a sliding mode control of dielectric charge," Microelectronics Reliability, vol. 55, no.9-10, pp. 1926-1931, 2015.

[29] M.T. Atienza and L. Kowalski and S. Gorreta and V. Jiménez and L.M. Castañer and M. Dominguez-Pumar, "Sliding mode analysis applied to improve the dynamical response of a spherical 3D wind sensor for Mars atmosphere", Sens. and Act. A: Physical, vol. 267, pp. 342-350, 2017.

[30] G. Sun and L. Wu and Z. Kuang and Z. Ma and L. Zhiqiang, "Practical tracking control of linear motor via fractional-order sliding mode", Automatica, vol. 94, pp. 221-235, 2018.

[31] H. Du and X. Chen and G. Wen and X. Yu and J. Lu, "Discrete-Time Fast Terminal Sliding Mode Control for Permanent Magnet Linear Motor", IEEE Trans. Ind. Electron, vol 65-12, pp. 9916-9927, 2018.

[32] N. Wang and S. Lv and W. Zhang and Z. Liu and M.J. Er, "Finitetime observer based accurate tracking control of a marine vehicle with complex unknowns", Ocean Engineering, vol. 145, pp. 406-415, 2017.

[33] N. Wang and S.F. Su and M. Han and W.H. Chen, "Back-propagating Constraints-Based Trajectory Tracking Control of a Quadrotor With Constrained Actuator Dynamics and Complex Unknowns", IEEE Trans. on Systems, Man and Cybernetics: Systems, 2018.

[34] G. Montseny "Diffusive Representation of Pseudo-differential Timeoperation," Proc. of ESAIM, vol. 5, pp. 4817-4828, 1998.

[35] M. Dominguez-Pumar, M.T. Atienza, L. Kowalski, S. Novio, S. Gorreta, V. Jimenez, and S. Silvestre, "Heat Flow Dynamics in Thermal Systems Described by Diffusive Representation," IEEE Trans. Ind. Electron, vol. 64, no.1, pp. 664-673, 2017.

[36] C. Bheesayagari, S. Gorreta, J. Pons-Nin and M. Dominguez-Pumar, "Second order sigma-delta control of charge trapping for MOS capacitors", Microelectronics Reliab., vol. 76-77, no. 4, pp. 635-639, 2017. 
[37] Hebertt Sira-Ramirez, Sliding Mode Control: The Delta-Sigma Modulation Approach. Switzerland: Birkhäuser-Springer, 2015.

[38] T.P. Ma and P.V. Dressendorfer, Ionizing Radiation Effects in MOS Devices and Circuits, NY, USA: Wiley, 1989. 